\title{
Enquête
}

Archives de la revue Enquête

5 | 1989

Biographie et cycle de vie

\section{Le cours de vie comme institution sociale}

\section{Martin Kohli}

\section{(2) OpenEdition}

\section{Journals}

Édition électronique

URL : http://journals.openedition.org/enquete/78

DOI : 10.4000/enquete.78

ISSN : 1953-809X

\section{Éditeur :}

Cercom, Éditions Parenthèses

\section{Édition imprimée}

Date de publication : 2 mars 1989

\section{Référence électronique}

Martin Kohli, «Le cours de vie comme institution sociale », Enquête [En ligne], 5 | 1989, mis en ligne le 27 juin 2013, consulté le 30 avril 2019. URL : http://journals.openedition.org/enquete/78 ; DOI :

10.4000/enquete.78

Ce document a été généré automatiquement le 30 avril 2019. 


\title{
Le cours de vie comme institution sociale
}

\author{
Martin Kohli
}

1 La démarche biographique doit tenir compte à la fois des questions de sociologie de structure et de sociologie d'action ou de sujet, comme cela a été bien exprimé par les organisateurs de ce colloque. En outre, cette démarche n'est pas seulement une démarche méthodique, mais aussi et surtout une démarche qui met en évidence certains «faits sociaux » : un certain type de règles qui organisent la vie sociale - on pourrait aussi dire un certain type de codes dont les sujets se servent pour construire la réalité. En essayant de reconstruire ces règles, j'ai été frappé par une impression qui peu à peu a pris des contours plus précis : c'est qu'il y a une évolution historique, une transformation qui a mené à un régime biographique beaucoup plus élaboré -ce que j'appelle $l^{\prime} \ll$ institutionnalisation du cours de vie $»^{1}$.

2 Il s'agit tout d'abord d'une collection de faits divers qui pourtant s'organisent en une structure, et il faut parler aussi bien de ces faits divers que de la structure. Je dois vous avertir que c'est une approche très globale, se référant à des époques larges (comme la «société moderne»), qui peut-être contraste avec la notion d'histoire de vie comme entamant un trajet historique spécifique et de courte durée. Par « institutionnalisation du cours de vie ", je désigne une unité contradictoire revêtant trois dimensions. La première est celle de la chronologie: il y a une normalisation (ou standardisation) du cours de vie dans le sens de l'évolution d'une séquence chronologique, avec des étapes bien définies. La seconde, est celle de la continuité: avec la création d'une vie prévisible et avec une mesure de sécurité (aussi au sens matériel). La troisième concerne la biographie propre : il s'agit de la genèse d'un code de développement personnel, de croissance (personal growth) - code qui dit que la vie est un projet personnel, et qu'il faut décider soi-même.

$3 \mathrm{Du}$ point de vue structurel, il faut relever deux aspects dans cette évolution. Premièrement, l'institutionnalisation du cours de vie a évolué autour de la transformation du système de travail. Donc, aujourd'hui nous avons une tripartition nette du cours de vie (pour les hommes d'abord, mais de plus en plus aussi pour les femmes), avec une période de préparation, une période d'activité et une période de retraite - une 
tripartition qui nous semble évidente mais qui est un produit historique assez nouveau. La transformation qui sert de base à cette évolution est le passage d'une économie de production domestique à une économie de marché, basée sur le travail formellement libre, travail contractuel, travail salarié. Ce fut une transformation assez lente d'ailleurs qui n'a été achevée que, disons, dans les années cinquante de ce siècle. Deuxièmement, l'institutionnalisation $\mathrm{du}$ cours de vie est étroitement liée au processus d'individualisation, à la création d'individus au sens moderne, c'est-à-dire la libération, ou le relâchement des sujets, des liens de famille et de localité. Il est important de voir que c'est un processus structural, non pas une dissolution du tissu social mais une transformation dans laquelle les individus deviennent les unités de base du tissu social.

4 Je vais donc vous raconter une histoire en deux étapes: d'abord, l'histoire d'une institutionnalisation progressive durant le siècle passé jusqu'à peu près la fin des années soixante de notre siècle; ensuite, l'histoire de l'arrêt ou même du renversement de ce processus, on pourrait dire de la désinstitutionnalisation qui est apparue au cours des vingt dernières années. Commençons par quelques exemples descriptifs de la première étape.

5 Premier exemple très simple, celui de la durée de la vie : on peut voir qu'il y a cent ans, un tiers des femmes vivaient jusqu'à 60 ans et un seizième jusqu'à quatre-vingts ans. Aujourd'hui, neuf femmes sur dix vivent jusqu'à 60 ans, et plus de la moitié à quatrevingts ans. La mortalité infantile a considérablement diminué, mais aussi celle des adolescents et des adultes d'âge " actif »; la plupart des gens vivent jusques vers 60 ans, puis meurent dans un intervalle d'âge assez étroit. (Les données sont celles de l'Allemagne, mais les autres pays de l'Ouest n'en sont pas loin). C'est un processus bien simple, tout d'abord une régularité statistique, mais qui relève aussi des projections biographiques: une transformation d'un régime de mort imprévisible à un régime de durée prévisible. Ce qui rend d'ailleurs la mort précoce beaucoup plus dure à supporter : elle est une rupture beaucoup plus marquée.

Deuxième exemple : le cycle familial. Il y a une régularisation des événements de la vie familiale : mariage - naissance du premier enfant - naissance du dernier enfant - passage des enfants à l'état adulte - et finalement mort du mari ou de la femme. Dans la famille «prémoderne » (européenne), ces événements n'étaient pas axés sur la chronologie de la vie individuelle, mais sur les nécessités de l'unité domestique qui était aussi l'unité de production. Il y avait une grande variance des âges de mariage, de grandes différences d'âge entre les enfants, et une large proportion de célibataires, célibataires forcés surtout, pour des raisons économiques. Jusqu'aux années 1960 on peut montrer dans tous les pays de l'Ouest que le célibat - sauf pour le personnel de l'Église catholique - a presque disparu, le mariage est devenu un événement qui s'insère comme point de repère normal dans les plans biographiques de tout le monde. L'âge de mariage, l'âge où on a les enfants - et par cela l'âge où les enfants quittent la famille paternelle - sont inscrits dans un intervalle beaucoup plus étroit. Donc, ce qu'on appelle le cycle familial est un produit de l'évolution historique, et il ne fait pas beaucoup de sens de parler d'un cycle pour la famille d'il y a cent cinquante ans.

7 Le troisième exemple est la construction légale et administrative de la démarcation par âges. Pour ce qui est de la « découverte sociale » de l'enfance et de la jeunesse comme âge de la vie propre, séparée de la vie adulte (comme Philippe Ariès l'a montré le premier), elle est évidemment normalisée et soumise à la chronologie par le système scolaire avec sa gradation très fine, et par des démarcations légales de toutes sortes : responsabilité civile, 
pénale, participation politique. Le code Napoléon fut d'ailleurs le premier à introduire des démarcations par âge précises. On trouve aussi une démarcation de l'âge avancé, de la vieillesse, par les systèmes de retraite. La vieillesse, dans le sens moderne - comme âge distinct du point de vue structurel et chronologique - est un produit assez moderne. Elle est basée sur le travail salarié ou plus précisément sur les systèmes publics d'assurances de vieillesse qui ont été créés comme réponse au problème du travail salarié. Parmi les hommes en Allemagne de plus de 70 ans, au début du siècle, la moitié était encore au travail, contre $3 \%$ de nos jours. La vieillesse est donc devenue une étape de la vie qui fait partie du cours normal : une étape dont la plupart des gens font l'expérience et qui par cela peut être anticipée - qui commence à un âge bien défini, et qui est démarquée du régime de travail par une forme de vie tout à fait différente. Les systèmes publics de sécurité ont essayé de couvrir les risques créés par l'évolution du travail «libre» (salarié) et ont ainsi contribué à créer un cours de la vie marqué par la continuité et la sécurité. En même temps, ils constituent un nouveau type de contrôle politique et social. Le cas de l'Allemagne - avec la première caisse de retraite publique et obligatoire - est particulièrement bien placé pour mettre en évidence ces deux aspects contradictoires. On peut trouver maintes expressions de Bismarck disant qu'un système de retraite, comme assurance matérielle avec laquelle les ouvriers peuvent compter pour la fin de leur vie, est la meilleure garantie pour les détourner du socialisme et de la révolution. Les plans pour ce système ont été dressés dans les années 1870, alors que la mémoire de la Commune était très vive. Quand il dut légitimer la création du système auprès des entrepreneurs, Bismarck expliqua que c'était de l'argent bien investi, car investi pour l'ordre social. De l'autre côté, c'est aussi la création d'un « citoyen social » qui ne dépend pas de la bienfaisance avec ses implications dégradantes, mais a droit à une certaine sécurité matérielle. Voici les deux aspects d'indépendance matérielle et de contrôle.

On peut penser ici à la discussion en anthropologie économique et histoire économique sur l'« enchâssement » de l'économie (« enbeddedness » selon le terme de Karl Polanyi). La plupart des chercheurs semblent être d'accord sur le fait que cette structure sociale autour du marché a disparu avec la transformation de l'économie moderne. C'est aussi la façon dont E. P. Thompson, l'historien du mouvement ouvrier en Angleterre argumente avec son concept d' "économie morale ». En termes marxistes, on pourrait dire c'est la transformation complète en marchandise ("commodification») non seulement des biens mais aussi de la force de travail. Je crois que c'est un accord prématuré. Il y a une nouvelle économie morale, centrée sur le travail et le travailleur; tandis que les biens ont été transformés en marchandise, le travail n'est pas mis complètement à la disposition des règles de marché, il est enchâssé dans de nouvelles structures sociales. Marcel Mauss a déjà noté à propos de l'assurance sociale que c'est un nouveau régime d'enchâssement, un régime de réciprocité qui fonctionne autour du marché.

9 Le quatrième exemple est l'évolution des codes biographiques. Les grandes études sur l'histoire des mentalités et du processus de civilisation relèvent un point commun important : la transformation d'un type de contrôle social externe en un contrôle interne, achevé par les individus eux-mêmes. Norbert Elias décrit la transformation d'une forme de vie spontanée et limitée par les contraintes extérieures seulement à une forme de vie basée sur l'intériorisation des contraintes, ce qui implique aussi d'ailleurs une contrainte à différer la gratification de ses besoins, donc à se gérer en perspective biographique de long terme. Max Weber met en évidence le même point, en marquant la différence entre le catholicisme médiéval et le calvinisme en ces termes : le catholicisme médiéval est celui 
de gens qui éthiquement « vivent de main en bouche » - après avoir commis des péchés, ils vont à la confession et sont relâchés pour des nouveaux péchés. Les calvinistes doivent gérer toute leur vie d'une façon «méthodique». Michel Foucault va dans la même direction. On pourrait aussi mentionner ici, l'étude d'Albert Hirschman, sur la redéfinition du concept de "passion » destructrice en terme d'« intérêt», contrôlé et rationalisé comme une précondition de l'évolution du capitalisme moderne.

Sur un autre plan cette individualisation s'exprime dans la littérature autobiographique. Le genre autobiographique subit une transition en Allemagne, vers 1800, d'une structure « analyste » à une structure " développementale ». Dans la première, la vie est organisée par un séquence d'événements extérieurs, historiques, ou du cycle saisonnier de production domestique ; dans la seconde, elle est organisée par un programme personnel, autour et par le moi. (C'est là aussi que surgit cette structure narrative que beaucoup prennent aujourd'hui pour naturelle : la téléologique linéaire.) Ce processus est lié au développement des sciences, surtout la psychologie. Les deux champs sont joints en une personne, Karl Philip Moritz, qui a écrit le premier «roman psychologique » au sens moderne, un roman autobiographique développemental (Anton Reiser ${ }^{2}$ ) et qui a été aussi l'éditeur d'un journal scientifique, le Magazin für Erfahrungsseelenkunde, consistant surtout en documents biographiques, qui est considéré aujourd'hui comme le premier journal de cette discipline.

11 Ceci pour les exemples. Les raisons structurelles de cette évolution ne peuvent pas être approfondies dans le cadre de cette communication. Mais il faut dire que c'est une évolution surprenante en termes théoriques. Si on considère que l'âge est un critère ascriptif, et non pas un critère de performance, il apparaît que son essor contredit toutes les théories de la modernisation. En tout cas, les démarcations par âge chronologique ne s'accordent pas très bien avec le système normatif universaliste des sociétés modernes. Aux États-Unis ceci s'est traduit par une législation opposée à la retraite obligatoire, le tribunal constitutionnel ayant reconnu que le fait d'être chassé du travail à un âge spécifique était une violation des droits fondamentaux. De même il existe une contradiction entre l'institutionnalisation $\mathrm{du}$ cours de vie comme séquence chronologique normale, et le code biographique qui met l'accent sur le développement de l'individu et la possibilité (et nécessité) de faire des choix. Là encore, le problème est mis en évidence par les formes littéraires, surtout par ce genre particulier de roman qui existe en Allemagne vers 1800, le "roman d'éducation». Dans ces constructions littéraires, le développement s'arrête quand la personne a atteint l'âge adulte, avec un équilibre plus ou moins satisfaisant entre besoins personnels et contraintes sociales, ou avec une fin tragique où l'individu se brise contre ces dernières. Dans les deux cas, l'histoire ne porte que sur les premières phases de la vie, et ne s'étend jamais jusqu'à la vieillesse.

La même " solution » est visible à propos des droits de choix personnel de métier, de lieu d'habitation et de partenaire de mariage. La garantie de ces droits est un des piliers de toute constitution moderne. Mais les règles sociales en limitent l'impact: on choisit une fois, au début de la vie adulte, et après on est obligé d'en subir les conséquences. Dans la perspective de Niklas Luhmann, le sens structurel de cette évolution est clair. Le droit de choisir son métier a comme fonction de mobiliser des motifs personnels au-delà de la faim, des contraintes et de l'obéissance - des motifs donc qui reposent sur une prise en charge de l'individu par lui-même. C'est aussi le modèle du mariage bourgeois. Comme Luhmann le montre, il a été inventé par le romantisme, qui a transféré le code de l'amour 
passionnel en code de mariage et l'a ainsi stabilisé. On pourrait ajouter que la passion a plutôt été liquidée ; quoi qu'il en soit, la « solution » d'un seul choix par vie, même si elle est fondée sur des motifs personnels, s'avère de plus en plus instable.

Avec cette dernière remarque, nous quittons le trajet de l'institutionnalisation pour parvenir à la deuxième étape de notre histoire. Depuis les années 1960, on peut en effet constater une certaine désinstitutionnalisation du cours de vie. Pour commencer avec les séquences chronologiques, il y a une désinstitutionnalisation très nette dans le cycle familial. L'âge du mariage et d'avoir des enfants devient moins standardisé - la variance s'accroît. Il y a de nouveau une proportion croissante de gens non mariés. Les tables démographiques de mariage montrent en Allemagne que le « risque » de se marier pour les cohortes nées en 1940 et 1950 retrouve le niveau du début du siècle, avec moins de $80 \%$ qui se marient. On pourrait dire que c'est un retour à l'état prémoderne. Mais cela n'est valable que pour les données de «comportement ». Ce phénomène est le même, il est produit par des raisons différentes. Ce ne sont plus les raisons économiques qui empêchent les gens de se marier, ce sont des choix personnels entre une vie avec enfant (s) et une vie sans enfant. En Allemagne, on estime que près de $30 \%$ des cohortes de 1960 n'auront pas d'enfants. C'est plus élevé que partout ailleurs, mais c'est un modèle qui commence à prendre forme aussi dans les autres pays de l'Ouest.

L'évolution est moins nette dans le champ du travail. Il y a une certaine flexibilisation de la trajectoire professionnelle, volontaire mais surtout involontaire, avec des périodes de chômage; il y a plus de travail à temps partiel, et des transitions plus étalées entre jeunesse et travail et entre travail et retraite. Mais c'est moins fort que ne le laisse penser la rhétorique de flexibilisation. Pour ce qui est de la transition à la retraite, ce n'est pas la flexibilité qui s'impose mais une transition de plus en plus avancée. On relâche les gens plus tôt, et par cela on conserve la structure tripartite du cours de vie.

Dans le champ du code biographique on assiste à un processus d'individualisation. Il commence à toucher aussi les groupes qui sont jusqu'ici restés à sa marge. Par exemple, les femmes se conçoivent de moins en moins comme membres d'une famille, donc d'un agrégat social, et de plus en plus comme individus propres. Le code se radicalise dans la dimension temporelle. On ne cesse plus de choisir et de se développer lorsqu'on a atteint l'âge adulte, on ne cesse de se développer jusqu'à la mort. À cela s'ajoute une expérience profonde de contingence, de "tout pourrait être différent », et donc de la nécessité de choisir.

16 Si ces tendances se confirmaient, le résultat en serait une conduite de vie radicalement subjective. Autrefois, le dynamisme du code biographique était contrôlé par la structure normale institutionnalisée du cours de vie. Aujourd'hui c'est l'individualité elle-même qui est institutionnalisée, au sens d'une recherche permanente de critères personnels. Autrement dit: ce qui est institutionnalisé n'est plus une structure de comportement mais une structure de réflexion. 


\section{NOTES}

1. En retenant la forme orale de cette communication, je renonce à citer les sources et œuvres spécifiques que j'emploie pour construire mon argumentation. Les lecteurs qui s'y intéressent peuvent se référer à mes textes suivants : «The world we forgot. An historical review of the life course », in V.W. Marshall, ed., Later life. The social psychology of aging, Beverly Hills, Sage, 1986, p. 271-303 ; « Gesellschaftszeit und Lebenszeit. Der Lebenslauf im Strukturwandel der Moderne », in J. Berger, ed., Die Moderne. Kontinuitäten und Zäsuren, Göttingen, Schwartz, 1986, p. 183-208 ( Soziale Welt ", 4); "Retirement and the moral economy. An historical interpretation of the German case », Journal of Aging Studies, 1, 1987, p. 125-144.

2. K. P. Moritz, Anton Reiser, ein psychologischer Roman, Berlin, F. Maurer, 1785-1794, 5 parties en 3 vol. $(\mathrm{NdlR})$ 